\title{
Early detection of evolving critical illness myopathy with muscle velocity recovery cycles
}

Tankisi $\mathrm{A}^{1}$, Pedersen $\mathrm{TH}^{2}$, Bostock $\mathrm{H}^{3}$, Z'Graggen $\mathrm{WJ}^{4}$, Larsen $\mathrm{LH}^{5}$, Meldgaard $\mathrm{M}^{5}$, Elkmann $\mathrm{T}^{1}$, Tankisi $\mathrm{H}^{5,6}$

${ }^{1}$ Department of Anaesthesiology and Intensive Care, Aarhus University Hospital, Aarhus, Denmark ${ }^{2}$ Department of Biomedicine, Aarhus University, Aarhus, Denmark.

${ }^{3}$ Institute of Neurology, University College London, Queen Square House, London, United Kingdom

${ }^{4}$ Departments of Neurology and Neurosurgery, Inselspital, Bern University Hospital, University of Bern, Bern, Switzerland.

${ }^{5}$ Department of Clinical Neurophysiology, Aarhus University Hospital, Aarhus, Denmark ${ }^{6}$ Institute of Clinical Medicine, Aarhus University, Aarhus, Denmark

\section{Corresponding author:}

Hatice Tankisi, MD, PhD

Aarhus University Hospital

Palle Juul-Jensens Boulevard 165,

DK-8200 Aarhus N, Denmark

Tel.: +4578454397 E-mail address: hatitank@rm.dk

Declarations of interest: Professor Hugh Bostock receives royalties from UCL for sales of his Qtrac software used in this study. The other authors have no conflicts of interest to disclose. All authors have approved the final paper.

Acknowledgments: This study was financially supported by Lundbeck Foundation (Grant number: R290-2018-751) and Independent Research Fund Denmark (Grant number: 9039-00272B). 


\section{Highlights}

- 24 patients with intensive care unit acquired weakness and 34 controls were examined with conventional electrophysiological methods and muscle velocity recovery cycles

- Muscle velocity recovery cycles showed $100 \%$ sensitivity and specificity in distinguishing patients from controls

- Muscle velocity recovery cycles can detect changes in patients with intensive care unit acquired weakness not yet fulfilling diagnostic criteria for critical illness myopathy. 


\begin{abstract}
Objective: To investigate the sensitivity of muscle velocity recovery cycles (MVRCs) for detecting altered membrane properties in critically ill patients, and to compare this to conventional nerve conduction studies (NCS) and quantitative electromyography (qEMG).
\end{abstract}

Methods: Twenty-four patients with intensive care unit acquired weakness (ICUAW) and 34 healthy subjects were prospectively recruited. In addition to NCS (median, ulnar, peroneal, tibial and sural nerves) and qEMG (biceps brachii, vastus medialis and anterior tibial muscles), MVRCs with frequency ramp were recorded from anterior tibial muscle.

Results: MVRC and frequency ramp parameters showed abnormal muscle fiber membrane properties with up to $100 \%$ sensitivity and specificity. qEMG showed myopathy in 15 patients (63\%) while polyneuropathy was seen in $3(13 \%)$. Decreased compound muscle action potential (CMAP) amplitude (up to 58\%) and absent F-waves (up to 75\%) were frequent, but long duration CMAPs were only seen in one patient with severe myopathy.

Conclusions: Altered muscle fiber membrane properties can be detected in patients with ICUAW not yet fulfilling diagnostic criteria for critical illness myopathy (CIM). MVRCs may therefore serve as a tool for early detection of evolving CIM.

Significance: CIM is often under-recognized by intensivists, and large-scale longitudinal studies are needed to determine its incidence and pathogenesis.

Keywords: Critical illness myopathy; Critical illness polyneuropathy; Muscle velocity recovery cycles; Nerve conduction studies; Electromyography; Intensive care unit acquired weakness 


\section{Introduction}

Critical limb and respiratory muscle weakness, referred to as intensive care unit (ICU) acquired weakness (ICUAW) is a common disabling condition in patients admitted to ICUs. Critical illness myopathy (CIM) and critical illness polyneuropathy (CIP) are very frequent causes of ICUAW, and diagnosis and differentiation are only possible with electrophysiological studies and muscle biopsy (Latronico and Bolton, 2011; Friedrich et al., 2015; Z’Graggen and Tankisi, 2020; Tankisi et al., 2020a). Different studies conducted by neurologists or neurophysiologists have indicated that a third of all patients at ICUs develop CIM, CIP or both (Latronico and Bolton, 2011; Bolton, 2005; Koch et al., 2014). Nevertheless, in everyday clinical activities CIM or CIP are often somewhat neglected and simply referred to as complications in critically ill patients or weaning failure in ICU literature (Boles et al, 2007; Maggiore et al, 2018) indicating that these conditions are not recognised satisfactorily by intensivists.

Currently the diagnosis of CIM and CIP is made by conventional nerve conduction studies (NCS), electromyography (EMG) and muscle biopsy showing preferential myosin loss (Llano-Diez et al., 2012; Friedrich et al., 2015; Swash and de Carvalho, 2020). However, these approaches detect advanced changes only, leaving patients, especially in the early stages, at risk of being missed. Reduced compound muscle action potential (CMAP) amplitude may be seen both in CIM and CIP, and sensory NCS, particularly examination of the sural nerve, is always difficult, and an absent response may be unreliable due to oedema, positioning of the patient or noise from equipment in ICU (Tankisi et al., 2020b). The existing indicators of CIM, such as prolonged CMAP duration, are based on small retrospective studies (Park et al., 2004; Goodman et al., 2009). So better techniques for early and accurate diagnosis are needed.

Several risk factors have been suggested for development of CIM and CIP including sepsis, multiple organ failure, hyperglycemia and immobilization (Swash and de Carvalho, 2020; Llano- 
Diez et al., 2012). These risk factors are suggested to cause muscle membrane depolarisation leading to inability of muscle fibres to generate electrical signals (loss of excitability), with ensuing muscle paralysis believed to be a main disease mechanism in CIM. Until recently, however, there has been no convenient method to measure such altered muscle membrane properties in patients. Bostock and Z'Graggen developed an automated method for recording of muscle velocity recovery cycles (MVRCs) that provide in vivo information about muscle membrane properties such as membrane potential (Z'Graggen and Bostock, 2009). The MVRCs are measured by direct muscle stimulation and electromyographic needle recording, and the method is fast and simple to perform and independent of patient's cooperation (Z'graggen and Bostock, 2009; Witt et al., 2020). The utility of MVRCs has been shown in muscle channelopathies (Tan et al., 2019; Boland-Freitas et al., 2018; Tan et al., 2018) and it has been used in showing in vivo evidence of altered membrane properties in neurogenic muscle fibres (Witt et al. , 2019) but so far, the use of MVRCs in CIM has been limited to one study of 10 patients with probable CIM by Z'Graggen and co-workers (Z'Graggen et al., 2011). The number of conditioning stimuli was limited to two in this study and the newer frequency ramp protocol was not applied.

Our aim in this study was to investigate the utility of MVRCs in detecting altered muscle membrane properties in a larger cohort of critically ill patients and with more advanced protocols. We also aimed to compare the sensitivity of conventional NCS and quantitative EMG (qEMG) with the MVRC technique.

\section{Material and Methods}

\subsection{Patients and healthy controls}

In this study, 24 patients with ICUAW at the Department of Anaesthesiology, Aarhus University Hospital were prospectively included between April 2017 and May 2020. ICUAW diagnosis requires: 1) a positive history for critical illness with multiorgan dysfunction and 2) clinical finding 
of limb weakness or difficulty in weaning the patient from the ventilator after exclusion of nonneuromuscular causes (Latronico and Bolton, 2011; Z’Graggen and Tankisi, 2020). All 24 patients in this study had weaning failure, defined as the failure to pass a spontaneous-breathing trial or the need for reintubation within 48 hours following extubation (Boles et al., 2007).

Exclusion criteria were a history of pre-existing myopathy or peripheral neuropathy and bleeding tendency. None of the patients received long-term treatment with neuromuscular blocking agents. All examinations were performed by the same examiner (HT) using conventional NCS and EMG as well as MVRCs. The patient charts were reviewed for survival in July 2020, after 2 months to 3 years interval from the neurophysiological investigation. The survival was noted as "yes" or "no" if the patient died by July 2020 and the number of survived days from electrophysiological examination to death, or to the date of review of patient files, was recorded.

The Ethics Committee of the Central Denmark Region approved this study. Written informed consent was obtained from the next of kin and an independent physician for inclusion in the study. We intended to subsequently ratify this by the patients if the patient became conscious before discharge, but this was not the case for any of the patients. Written consent was obtained from the healthy subjects as well. The data used in this study has also been approved by the Danish Data Protection Agency.

The patient results were compared with 34 age and sex-matched healthy controls or to the laboratory's normal material.

\subsection{Clinical examination}

All patients underwent a detailed clinical evaluation. Deep tendon reflexes were examined in all patients and force measurements were made if possible since all patients were in mechanical ventilation. Organ failure was assessed using the "Sequential Organ Failure Assessment" (SOFA) scoring scheme (Vincent et al., 2000) on the day of electrophysiological examinations. 
Additionally, the ICU scoring system for predicting mortality "Simplified Acute Phyiology" (SAPP III) score (Moreno et al., 2005), assessed by the anaesthesiologist at admission to ICU was noted.

\subsection{Laboratory examinations}

Laboratory examinations were performed within 2 hours of the electrophysiological investigations. Laboratory examinations included plasma levels of sodium, potassium, calcium, chloride, creatinine and blood gas analysis (pH, bicarbonate, $\mathrm{pO} 2, \mathrm{pCO} 2$ and base excess).

\subsection{Conventional electrophysiological studies}

All studies were done using Keypoint.Net EMG equipment (Dantec, Skovlunde, Denmark) with conventional methods (Stålberg et al, 2019; Tankisi et al., 2019). For NCS, disposable pre-gelled surface electrodes $(\mathrm{Ag} / \mathrm{AgCl})$ with a recording area of $15 \mathrm{~mm}$ x $20 \mathrm{~mm}$ were used. The results were compared to laboratory control material. Skin temperature was maintained over $32^{\circ} \mathrm{C}$ by a heating lamp or a hair dryer.

In all patients, NCS were performed as described below, and EMG was recorded from biceps brachii, anterior tibial and vastus medialis muscles. In healthy subjects, EMG was only done in anterior tibial muscle. The patients ${ }^{\prime}$ NCS results and EMG of biceps brachii and vastus medialis were compared with laboratory normal material, while EMG of anterior tibial muscle was compared with the 34 healthy subjects.

\section{Median and ulnar sensory and motor NCS}

For median sensory NCS, the nerve was stimulated at the wrist and antidromic sensory nerve action potential (SNAP) was recorded from digit II. If median nerve was abnormal, ulnar nerve was also examined by stimulating at wrist and recording from the fifth digit. If there were signs of carpal tunnel syndrome, ulnar NCS results were used for analyses. For motor NCS, median CMAP was 
recorded from abductor pollicis brevis and ulnar CMAP from abductor digiti minimi. Median nerve was stimulated at wrist and at the elbow, and ulnar nerve at the wrist and below elbow.

\section{Peroneal and Tibial Motor NCS}

For motor NCS of the tibial nerve, the nerve was stimulated behind the medial malleolus and at popliteal fossa, and the recording electrodes were placed on the abductor hallucis muscle. For peroneal nerve, the stimulation sites were ankle, capitulum fibulae and fossa poplitea and the recording electrodes were placed over the extensor digitorum brevis and anterior tibial muscles.

\section{Sural NCS}

For the NCS of the sural nerve, the surface electrodes were placed behind the lateral malleolus, and the nerve was stimulated at the sura lateral to the edge of the Achilles tendon at a distance of $13 \mathrm{~cm}$ proximal to the recording electrode. NCS of the distal segment of sural nerve was done by recording from the mid-portion of the fifth metatarsal bone, just lateral to the extensor digitorum longus tendon of the fifth toe. Stimulation site was posterior to the lateral malleolus.

The evaluated motor NCS parameters were distal motor latency, motor and sensory conduction velocity (CV), CMAP and SNAP amplitude, CMAP duration and minimum F-wave latency. Peakto peak amplitude was used for all motor NCS and for sural NCS, while base-to-peak amplitude was used for median and ulnar sensory NCS measurements. CMAP duration was measured from the first negative deflexion to the next crossing of the baseline.

\section{CMAP ratio (neCMAP/mCMAP)}

For the anterior tibial muscle, the ratio obtained from nerve stimulation CMAP (neCMAP) to direct muscle stimulation CMAP (mCMAP) was calculated (Latronico et al., 2005, 2009; Rich et al., 1997). Direct muscle stimulation was done with a monopolar needle electrode serving as a cathode (25 mm x 26G, TECA) inserted perpendicularly into the distal half of the muscle away from the neuromuscular endplate while a non-polarizable surface electrode served as anode, placed 1-2 cm 
distally. Recordings were made with a concentric $35 \mathrm{~mm}$ needle electrode (Medtronic, Skovlunde, Denmark) to obtain supramaximal mCMAP. This electrode was inserted into the anterior tibial muscle approximately $50 \mathrm{~mm}$ proximal to the stimulation cathode. For nCMAP recordings, the peroneal nerve was stimulated below capitulum fibulae and the supramaximal CMAP was measured using the same recording electrode as for direct muscle stimulation. nCMAP/mCMAP ratio analysis was applied first after the recruitment of the first 6 patients.

\section{Repetitive Nerve Stimulation}

To screen for neuromuscular transmission defects, the median nerve was stimulated at the wrist 10 times at $3 \mathrm{~Hz}$ while recording from the abductor pollicis brevis muscle. A decrease of more than $10 \%$ in amplitude for the 5 th stimulation compared to the 1 st stimulation was considered as abnormal (Stålberg et al., 2019).

\section{Quantitative Electromyography (qEMG)}

In all patients, EMG of biceps brachii, anterior tibial and vastus medialis muscles was performed using a concentric $35 \mathrm{~mm}$ Dantec needle electrode. The Department's standard filter settings of 20 $\mathrm{Hz}-10 \mathrm{kHz}$, gain (100 mV/division) and sweep speed (10 ms/division) were used.

The presence of spontaneous activity, i.e. fibrillation potentials (fibs), positive sharp waves (psws), fasciculations and complex repetitive discharges was assessed at 10 separate sites, and spontaneous activity at more than two sites was regarded as abnormal (Tankisi et al., 2007). Quantitative EMG using individual MUP analysis (Buchthal, 1962) was done by sampling of 20 potentials during passive movements of the elbow, knee and ankle or by scratching the foot sole with the naked end of a cotton swab for anterior tibial muscle. Mean duration, amplitude and percentage of polyphasic potentials were evaluated. A MUP has been described as polyphasic if number of phases were $\geq 5$ (Stålberg et al., 2019). If the number of polyphasic MUPs was $\geq 3$ out of 20 MUPs, the incidence of polyphasic potentials was interpreted as abnormal and the mean duration was calculated for the 
simple potentials. A MUP duration more than $20 \%$ below the mean of laboratory controls, which corresponds to the $95 \%$ confidence interval, was considered as myopathic.

\subsection{Muscle Velocity Recovery Cycles (MVRCs)}

All tests were performed in all patients and healthy controls. Recordings were controlled by the M3REC3.QRP protocol and the QtracS component of the QtracW software (written by H. Bostock, copyright Institute of Neurology, University College London, UK). The recording set-up included an isolated constant current stimulator (DS5; Digitimer Ltd), a HumBug $50 \mathrm{~Hz}$ noise eliminator and a D440 amplifier (Digitimer Ltd).

Examinations included 1) MVRC recordings at rest, and 2) Frequency Ramp protocol, with intermittent repetitive stimulation at frequencies ramped from 1 to $30 \mathrm{~Hz}$.

Both examinations were performed in anterior tibial muscle by direct muscle stimulation of a column of muscle fibers and measuring the response from the same fibers. Stimulation was done with a monopolar needle electrode serving as a cathode (25 mm x 26G, TECA) inserted perpendicularly into the distal half of the muscle away from the neuromuscular endplate. Stimuli (0.05-ms rectangular current pulses) were delivered through this needle. A non-polarizable surface electrode serving as an anode was placed approx. $1 \mathrm{~cm}$ distal to the cathode. The recording electrode was a concentric needle electrode (25 mm x 30G, Dantec) inserted perpendicularly $20 \mathrm{~mm}$ proximal to the stimulating monopolar needle. A ground electrode was placed distal to both needles. The needle electrodes were adjusted to obtain a stable negative peak response by stimulation of less than $10 \mathrm{~mA}$. The stimulation current was not changed during recordings after this adjustment.

MVRCs were recorded using a protocol that had 1, 2 or 5 conditioning stimuli followed by a test stimulus from which the MVRC was measured. The duration between the last conditioning stimulus and the test stimulus is referred to as the inter-stimulus interval (ISI) and this was decreasing in 34 
steps from $1000 \mathrm{~ms}$ to $1.4 \mathrm{~ms}$. Latencies were recorded from the applied test stimulus to the peaks of the responses from the muscle fibers. Recovery cycles were plotted as percentage latency change, in the form of conduction velocity slowing (\%), as a function of ISI (logarithmic scale). The evaluated MVRC parameters were muscle relative refractory period (MRRP), early, late and residual supernormalities.

For the frequency ramp protocol, a $1 \mathrm{~s}$ train of stimuli was delivered every $2 \mathrm{~s}$. The number of stimuli in the train was increased by 1 from 2 to 31 stimuli, so that the mean stimulation rate was ramped up from 1 to $15.5 \mathrm{~Hz}$ over 1 minute. Responses were measured to the first and last stimuli in each train. Stimulus cycles with the test stimulus alone were recorded before and after the end of the frequency ramp with 10 and 15 cycles, respectively and each at $0.5 \mathrm{~Hz}$. The evaluated frequency ramp parameters were $\operatorname{Lat}(15 \mathrm{~Hz}) \%$ and $\mathrm{Latf}(15 \mathrm{~Hz}) \%$ (latency to the first and last response in train at $15 \mathrm{~Hz}$ ), $\operatorname{Latf}(30 \mathrm{~Hz}) \%$ (latency to the first response in train at $30 \mathrm{~Hz}$ ) and $\operatorname{Pkf}(15 \mathrm{~Hz}) \%$ and $\mathrm{Pkf}(30 \mathrm{~Hz}) \%$ (amplitude to the first stimuli at $15 \mathrm{~Hz}$ and $30 \mathrm{~Hz}$ trains, respectively).

\subsection{Data analysis}

The QtracP component of the QtracW software was used for the statistical analyses and for generating figures. Variables were tested with students' t-test or Mann-Whitney U-test depending on whether the data was normally distributed using Lilliefors test. Correlation analyses were performed by Pearson's correlation coefficient or Spearman's rank. The ability of MVRCs and MUP analysis results in anterior tibial muscle to discriminate patients from healthy controls was evaluated with receiver operating characteristic (ROC) analyses, by determining the area under the curve (AUC). Sensitivity, specificity and accuracy (average of sensitivity and specificity) were calculated using ROC curves. Results with $\mathrm{P}<0.05$ were considered significant.

\section{Results}

\subsection{Participant demographics}


There was no significant difference in age between healthy controls (mean: $58.6 \pm 14$ ) and patients (mean: $64.9 \pm 12$ ) or in gender (healthy controls: 15 females, 19 males and patients: 10 females, 14 males). At the timepoint of neurophysiological examination, all patients were mechanically ventilated and most of them were sedated to tolerate this and none of them could cooperate for a full force measurement. None of the patients could therefore move the joints against resistance (MRC <4). Deep tendon reflexes were reduced or absent in all patients. The mean Glasgow Coma Scale (GCS) was $8.4 \pm 2.3$ (range: $5-12$ ) and the SOFA score was $9.7 \pm 3.5$ (range: $6-17$ ). The diagnosis, SAPP III Score at the admission, survival from ICU stay, days after ICU admission and SOFA score on the electrophysiological examination day for each patient are shown in Table 1. Of the 24 patients, 7 (29\%) survived the ICU stay. Of these, 3 patients (patients \#3, \#4 and 16) died 4 months2 years after discharge from ICU because of sudden unexpected death in epilepsy (SUDEP), intracranial bleeding and cardiac insufficiency. The number of survival days ranged from 17 to 910 days (median: 38.5 days).

\subsection{Conventional NCS and EMG}

Conventional EMG and NCS results are summarized in Table 1. Fibs/psws were seen in 5 patients while 4 patients had complex repetitive discharges in one or more muscles. In all patients, it was possible to collect 20 MUPs in biceps brachii while in anterior tibial muscle quantitative MUP analysis could be performed in 22 patients. In vastus medialis this could only be done in 3 patients. In 15 patients (63\%), MUP analysis showed myopathic changes with short duration in biceps brachii (Fig. 1) with (50\%) or without (50\%) increased amount of polyphasic potentials compared to laboratory's healthy controls. Of these, there was also myopathic MUPs in 2 of the 3 vastus medialis muscles from which MUPs could be obtained. In anterior tibial muscle, 6 of 22 patients (27\%) showed MUPs outside 95\% confidence interval with decreased duration compared to healthy controls (Table 1, Fig. 2). In all patients with myopathic MUPs in anterior tibial muscle, biceps 
brachii was also myopathic. Overall, 15 patients (63\%) had myopathy in EMG fulfilling the criteria for probably CIM.

There were 3 patients (13\%) who showed sensory and motor NCS abnormalities with reduced SNAP and CMAP amplitudes, and decreased motor CVs consistent with polyneuropathy. These 3 patients also had myopathy. None of the other 19 patients had decreased CV or prolonged latencies in any of the nerves. However, reduced CMAP amplitudes and absent F-waves were very often seen in all nerves. With median nerve stimulation, CMAP amplitude was reduced in 11 patients, F-waves were absent in 10 but CMAP duration was prolonged (12.3ms) only in one patient who had severe myopathic findings (\#19) (Fig. 2). In the tibial nerve, CMAP amplitude was reduced in 13 patients (54\%), F-waves were absent in 13 patients and CMAP duration was prolonged in 5 patients (21\%). Similarly, in the peroneal nerve, reduced CMAP amplitude (14 patients (58\%)) and absent F-waves (18 patients $(75 \%)$ ) were common but prolonged CMAP duration was not as common (7 patients (29\%)) (Table 1). In 18 patients neCMAP/mCMAP analysis was performed and all had a ratio $\geq 0.5$ including the 2 patients (\#15 and \#21) with both polyneuropathy and myopathy findings.

Table 1. Patient demographics and results of quantitative electromyography and nerve conduction studies.

\begin{tabular}{|c|c|c|c|c|c|c|c|c|c|c|c|c|c|c|c|c|c|c|}
\hline \multirow[t]{2}{*}{$I D$} & \multirow[t]{2}{*}{$\begin{array}{l}\text { Age/ } \\
\text { Sex }\end{array}$} & \multirow[t]{2}{*}{$\begin{array}{c}\text { Diagnosis } \\
\text { (admission) }\end{array}$} & \multirow[t]{2}{*}{ Survival } & \multirow[t]{2}{*}{$\begin{array}{l}\text { SAPP } \\
\text { score }^{\epsilon}\end{array}$} & \multirow[t]{2}{*}{$\begin{array}{l}I C U \\
\text { stay }^{\$}\end{array}$} & \multirow[t]{2}{*}{$\begin{array}{l}\text { SOFA } \\
\text { score }\end{array}$} & \multicolumn{3}{|c|}{$\begin{array}{l}q E M G(S A \text { and } \\
M U P \text { analysis })\end{array}$} & \multicolumn{3}{|c|}{$\begin{array}{l}\text { Median or ulnar } \\
\text { NCS }\end{array}$} & \multicolumn{2}{|c|}{ Tibial NCS } & \multicolumn{2}{|c|}{ Peroneal NCS } & \multirow{2}{*}{$\begin{array}{l}\text { Sural } \\
\text { SNAP } \\
\text { Amp } \\
(\mu V)\end{array}$} & \multirow{2}{*}{$\begin{array}{l}\text { CMAP } \\
\text { ratio } \\
\text { nervel } \\
\text { muscle }\end{array}$} \\
\hline & & & & & & & $\begin{array}{c}B B \\
(m s)\end{array}$ & $\begin{array}{c}T A \\
(m s)\end{array}$ & $\begin{array}{c}V M \\
(m s)\end{array}$ & $\begin{array}{c}C M A P \\
\text { Amp } \\
(m V)\end{array}$ & $\begin{array}{c}C M A P \\
\text { Dur } \\
(m s)\end{array}$ & $\begin{array}{l}\text { SNAP } \\
A m p \\
(\mu V)\end{array}$ & $\begin{array}{l}\text { CMAP } \\
A m p \\
(m V)\end{array}$ & $\begin{array}{c}\text { CMAP } \\
\text { Dur } \\
(m s)\end{array}$ & $\begin{array}{c}\text { CMAP } \\
\text { Amp } \\
(m V)\end{array}$ & $\begin{array}{c}\text { CMAP } \\
\text { Dur } \\
(m V)\end{array}$ & & \\
\hline 1 & $71 / \mathrm{M}$ & Pneumonia & $\mathrm{N}$ & 64 & 7 & 8 & 12.2 & 12.6 & NA & 6.7 & 5.0 & 9.9 & 6.4 & 5.4 & 2.9 & 5.6 & 6.2 & $\mathrm{NE}$ \\
\hline 3 & $48 / \mathrm{M}$ & Cerebral abscess & $\mathrm{Y}$ & 72 & 19 & 11 & 8.8 & 8.7 & NA & 13.8 & 5.9 & 14.8 & 14.7 & 6.5 & 5.4 & 6.5 & 23.6 & $\mathrm{NE}$ \\
\hline 4 & $53 / \mathrm{M}$ & Hepatitis & $\mathrm{Y}$ & 83 & 15 & 14 & 7.8 & 10.6 & NA & $\underline{3.4 £}$ & $6.8 £$ & $3.0 £$ & $\underline{3.0}$ & 7.6 & 2.4 & 6.9 & NR & $\mathrm{NE}$ \\
\hline 5 & 63/M & Oesophagus op. & $\mathrm{Y}$ & 48 & 43 & 6 & 13.8 & 15.1 & 12.9 & 8.8 & 5.1 & 10.1 & 9.4 & 7.0 & 4.2 & 5.8 & 6.9 & $\mathrm{NE}$ \\
\hline 6 & $68 / \mathrm{F}$ & GIS tm. op. & $\mathrm{N}$ & 67 & 10 & 11 & 7.8 & 10.5 & NA & 3.7 & 6.0 & 12.9 & $\underline{4.3}$ & 6.9 & $\underline{0.8}$ & 6.4 & 3.5 & $\mathrm{NE}$ \\
\hline 7 & $76 / F$ & $\mathrm{ICH}$ & $\mathrm{N}$ & 43 & 15 & 8 & 8.0\# & 11.9\# & NA\# & 4.4 & 6.1 & 7.8 & 1.2 & 5.7 & $\underline{2.0}$ & 6.5 & 12.1 & 0.6 \\
\hline 8 & $70 / \mathrm{M}$ & Peritonitis & $\mathrm{N}$ & 69 & 16 & 7 & 9.8 & 11.3 & 9.9 & 1.9 & 5.9 & 15.3 & 4.9 & 5.5 & 0.1 & 7.6 & 3.3 & 1 \\
\hline 9 & $74 / \mathrm{M}$ & Ileus op. & $\mathrm{N}$ & 60 & 21 & 7 & 10.1 & 11.3 & NA & 6.1 & 5.4 & 7.3 & 6.4 & 5.6 & $\underline{0.8}$ & 7.1 & 6.3 & 0.9 \\
\hline
\end{tabular}




\begin{tabular}{|c|c|c|c|c|c|c|c|c|c|c|c|c|c|c|c|c|c|c|}
\hline 10 & $73 / \mathrm{F}$ & Encephalitis & $\mathrm{N}$ & 40 & 8 & 10 & 10.7 & \begin{tabular}{|l|}
13.7 \\
\end{tabular} & NA & 5.7 & 5.3 & 10.2 & 10.4 & 5.1 & 1.5 & 5.2 & 8.2 & 0.7 \\
\hline 11 & $63 / \mathrm{M}$ & $\mathrm{ICH}$ & $\mathrm{N}$ & 63 & 18 & 8 & 9.7\# & $12.6 \#$ & NA\# & $\underline{4.3}$ & 5.1 & 8.2 & $\underline{4.8}$ & 7.4 & $\underline{3.6}$ & 6.1 & 5.9 & 0.8 \\
\hline 12 & 69/F & Pancreatitis & $\mathrm{N}$ & 56 & 45 & 9 & 9.7 & 11.6 & 9.0 & $6.5 £$ & $5.7 £$ & $9.5 £$ & $\underline{3.4}$ & 8.1 & $\underline{1.1}$ & 7.5 & 5.1 & 1 \\
\hline 13 & $70 / \mathrm{F}$ & Stroke & $\mathrm{N}$ & 56 & 32 & 7 & 9.3* & \begin{tabular}{|l|}
11.4 \\
\end{tabular} & NA & 7.8 & 4.8 & 9.5 & $\underline{0.7}$ & 8.6 & $\underline{0.7}$ & 8.4 & 7.2 & 0.8 \\
\hline 14 & $33 / \mathrm{M}$ & $\mathrm{ICH}$ & $\mathrm{Y}$ & 61 & 37 & 6 & 12.3 & 12.6 & NA & 14.6 & 5.5 & 12.9 & 20.8 & 6.1 & 6.9 & 5.4 & 13.2 & 1 \\
\hline 15 & $64 / \mathrm{M}$ & $\begin{array}{l}\text { Heart op. ileum } \\
\text { ischemia }\end{array}$ & $\mathrm{N}$ & 51 & 28 & 16 & 9.8\# & NA\# & NA\# & $\underline{4.0} £$ & $6.9 £$ & NR $£$ & $\underline{0.6}$ & 5.3 & $\underline{0.1}$ & 6.2 & NR & 0.7 \\
\hline 16 & $70 / \mathrm{F}$ & Mamma ca. & $\mathrm{Y}$ & 92 & 10 & 8 & 11.6 & 12.3 & NA & 6.0 & 5.9 & 7.7 & 6.1 & 5.6 & 2.8 & 6.3 & 4.5 & 1 \\
\hline 17 & $70 / \mathrm{F}$ & Stroke & $\mathrm{N}$ & 73 & 9 & 15 & 10.5 & 12.8 & NA & $\underline{3.2}$ & 4.9 & 13.5 & $\underline{6.8}$ & 5.9 & 1.2 & 5.5 & $2.9 \S$ & 1 \\
\hline 18 & $78 / \mathrm{M}$ & Pneumonia & $\mathrm{N}$ & 76 & 12 & 10 & $11.7 *$ & 13.6 & NA & $3.0 £$ & $6.5 £$ & $4.1 £$ & $\underline{3.3}$ & 5.6 & NR & NR & 3.2 & 1 \\
\hline 19 & $54 / \mathrm{F}$ & Ileus & $\mathrm{N}$ & 62 & 21 & 17 & $\begin{array}{l}8.3 \# \\
\end{array}$ & NA\# & NA\# & $\underline{0.4}$ & 12.3 & 43.1 & $\underline{1.3}$ & 9.8 & NR & NR & $3.4 \S$ & 0.9 \\
\hline 20 & 66/F & Meningitis & $\mathrm{Y}$ & 49 & 12 & 6 & $8.5^{*}$ & 9.5 & NA & 3.0 & 5.6 & 11.0 & 11.7 & 6.9 & 1.5 & 7.6 & 9.7 & 0.8 \\
\hline 21 & $64 / \mathrm{M}$ & Pancreatitis & $\mathrm{N}$ & 59 & 47 & 7 & 9.4 & 11.0 & NA & 3.9 & 4.4 & 2.7 & 1.7 & 4.8 & $\underline{0.1}$ & 5.7 & NR & 1 \\
\hline 22 & $71 / \mathrm{F}$ & Sialadenitis & $\mathrm{N}$ & 47 & 10 & 7 & $11.6^{*}$ & 13.2 & NA & 6.6 & 5.6 & 9.3 & 9.4 & 5.7 & 2.9 & 6.5 & 5.5 & 0.9 \\
\hline 23 & $79 / \mathrm{M}$ & GIS bleeding & $\mathrm{N}$ & 60 & 38 & 9 & 9.6 & 14.5 & NA & 1.8 & $\underline{6.1}$ & 11.9 & $\underline{1.3}$ & 9.7 & $\underline{0.4}$ & 8.9 & 3.4 & 0.8 \\
\hline 24 & $73 / \mathrm{M}$ & Anal ca. op & $\mathrm{N}$ & 65 & 16 & 7 & 9.8 & 10.2 & NA & 5.9 & 5.1 & 7.7 & 11.4 & 5.3 & 0.4 & 5.9 & 4.0 & 1 \\
\hline
\end{tabular}

SAPP: Simplified acute physiology, SOFA: Sequential organ failure assessment, ICU: Intensive care unit, qEMG: Quantitative electromyography, NCS: Nerve conduction studies, SA: Spontaneous activity, MUP: Motor unit potential, CMAP: Compound muscle action potential, SNAP: Sensory nerve action potential, BB: Biceps brachii, TA: Tibialis anterior, VM: Vastus medialis, NA: Not applicable, NE: Not examined, NR: No response, ca: Cancer, op: Operation, GIS: Gastrointestinal system, ICH: Intracerebral hemorrhage. Bold numbers indicate abnormal values and underlined numbers indicate the nerves with absent F-waves, *complex repetitive discharges, "fibrillation and/or positive sharp waves $>2$ sites, ${ }^{€}$ SAPP III score at admission, ${ }^{\$}$ ICU duration from admission to electrophysiological examination, ${ }^{\mathfrak{f}}$ ulnar nerve results instead of median nerve due to carpal tunnel syndrome, ${ }^{\S}$ distal sural NCS in addition to conventional sural NCS.

\subsection{Muscle Velocity Recovery Cycles (MVRCs)}

MUP analysis, in anterior tibial muscle, NCS and MVRC and frequency ramp results are shown in Table 2 and Figures 3 and 4. MUP duration was significantly shorter in patients compared with healthy controls but the MUP amplitudes were not different. CMAP amplitude was significantly decreased in patients compared with healthy controls but there was no difference in CMAP duration. When comparing the MVRC parameters, all parameters including muscle relative refractory period (MRRP), early supernormality and late supernormality measurements differed highly significantly between patients and healthy controls. The most significant two parameters, MRRP and late supernormality with 5 conditioning stimuli could differentiate all patients from 
healthy controls with $100 \%$ sensitivity and specificity (AUC: 1.000 ) while the sensitivity, specificity and AUC were lower for MUP parameters, CMAP amplitude and duration (Table 2, Fig. 3). Similarly, for frequency ramp measurements, the latency of the first response from trains at both 15 and $30 \mathrm{~Hz}$ differed highly significantly between the patients and healthy controls with high sensitivity and specificity whereas differences for amplitude measurements were less pronounced.

Table 2. Motor unit potential, NCS, MVRC and frequency ramp results in anterior tibial muscle in healthy controls and patients.

\begin{tabular}{|c|c|c|c|c|c|c|c|}
\hline & \multicolumn{2}{|c|}{ Mean $( \pm$ SE) } & \multirow{2}{*}{$\begin{array}{l}\text { Controls } \\
\text { vs } \\
\text { patients } \\
\text { p-value }\end{array}$} & \multirow[t]{2}{*}{$A U C$} & \multirow[t]{2}{*}{ Sensitivity } & \multirow[t]{2}{*}{ Specificity } & \multirow[t]{2}{*}{ Accuracy } \\
\hline & $\begin{array}{l}\text { Healthy controls } \\
(\mathrm{n}=34)\end{array}$ & Patients $(n=24)$ & & & & & \\
\hline MUP duration (ms) & $13.71 \pm 0.20$ & $12.08 \pm 0.36$ & $1.2^{-4}$ & 0.785 & 76 & 74 & 75 \\
\hline MUP amplitude $(\mu \mathrm{V})$ & $359.6 \pm 14.5$ & $315.5 \pm 16.8$ & 0.05 & 0.622 & 91 & 35 & 56 \\
\hline CMAP amplitude & $11.6 \times / \div 0.35$ & $6.3 \times / \div 0.83$ & $4.5^{-7}$ & 0.871 & 87 & 74 & 79 \\
\hline CMAP duration & $12.77 \pm 0.32$ & $12.75 \pm 0.31$ & 0.92 & 0.511 & 83 & 35 & 54 \\
\hline MRRP (ms) & $3.43 \pm 0.06$ & $6.53 \pm 0.46$ & $4.4^{-10}$ & 1.000 & 100 & 100 & 100 \\
\hline MSuperN $(<15) \%$ & $11.73 \pm 0.34$ & $4.56 \pm 0.62$ & $1.4^{-13}$ & 0.960 & 92 & 100 & 97 \\
\hline M5SuperN $(<15) \%$ & $14.0 \pm 0.42$ & $4.25 \pm 0.62$ & $8.6^{-17}$ & 0.989 & 96 & 100 & 98 \\
\hline MSuperN@(ms) & $7.3 \times / \div 0.21$ & $21.34 \times / \div 5.2$ & $3.0^{-11}$ & 0.956 & 83 & 97 & 91 \\
\hline MLSuperN(50-150)\% & $3.88 \pm 0.21$ & $1.91 \pm 0.27$ & $1.1^{-6}$ & 0.878 & 83 & 88 & 88 \\
\hline MXLSuperN(50-150)\% & $2.85 \pm 0.12$ & $1.01 \pm 0.14$ & $1.6^{-12}$ & 0.980 & 88 & 97 & 93 \\
\hline MX5LSuperN(50-150)\% & $8.10 \pm 0.23$ & $2.75 \pm 0.33$ & $5.7^{-17}$ & 1.000 & 100 & 100 & 100 \\
\hline MRSuperN(950)\% & $0.14 \pm 0.03$ & $0.01 \pm 0.08$ & 0.06 & 0.578 & 29 & 100 & 71 \\
\hline MX5RSuperN(950)\% & $1.18 \pm 0.09$ & $0.56 \pm 0.07$ & $6.4^{-6}$ & 0.854 & 83 & 79 & 81 \\
\hline Peak $(\mathrm{mV})$ & $2.13 \times / \div 0.20$ & $0.58 \times / \div 0.090$ & $2.3^{-9}$ & 0.919 & 92 & 79 & 85 \\
\hline Lat $(15 \mathrm{~Hz}) \%$ & $84.08 \pm 0.45$ & $96.40 \pm 1.03$ & $3.7^{-15}$ & 0.986 & 96 & 100 & 98 \\
\hline Latf $(15 \mathrm{~Hz}) \%$ & $94.52 \pm 0.31$ & $100.1 \pm 0.48$ & $8.1^{-13}$ & 0.982 & 92 & 100 & 97 \\
\hline $\operatorname{Pkf}(15 \mathrm{~Hz}) \%$ & $106.0 \pm 2.09$ & $99.12 \pm 2.81$ & 0.04 & 0.708 & 67 & 77 & 72 \\
\hline Latf(30Hz)\% & $95.88 \times / \div 0.43$ & $106.1 \times / \div 1.36$ & $5.5^{-12}$ & 0.963 & 96 & 88 & 91 \\
\hline $\operatorname{Pkf}(30 \mathrm{~Hz}) \%$ & $103.3 \pm 3.3$ & $84.95 \pm 3.93$ & $7.7^{-4}$ & 0.754 & 92 & 59 & 72 \\
\hline
\end{tabular}

NCS: Nerve conduction studies, MVRC: Muscle velocity recovery cycle, AUC: Area under the curve, MUP: Motor unit potential, CMAP: Compound muscle action potential, MRRP: Muscle relative refractory period. MSuperN $(<X)$ : Early supernormality at inter stimulus intervals below $15 \mathrm{~ms}$. M5SuperN: Supernormality with 5 conditioning stimuli. MLSuperN: Late supernormality. MXLSuperN: Extra late supernormality. MX5LSuperN: Extra late supernormality after 5 conditioning stimuli. MRSuperN: Residual Supernormality. MX5RSuperN: 
Residual supernormality after 5 conditioning stimuli. Lat $(\mathrm{XHz})$ : Latency of last response in train of stimuli at $\mathrm{X}$ $\mathrm{Hz}$ relative to unconditioned. Latf: Latency of first response in train of stimuli relative to unconditioned. Pkf: Peak of first response in a train of stimuli relative to unconditioned. The data are expressed as mean \pm SEM, except where the data are log-normally distributed in which case the data are shown as geometric mean $\times / \div$ geometric SEM (expressed as a factor). Bold numbers indicate significant $\mathrm{p}$ values.

\subsection{Correlations between MVRC parameters, qEMG, clinical scores and blood tests}

In healthy subjects, none of the MVRC or frequency ramp parameters correlated with MUP duration or amplitude $(\mathrm{p}>0.05)$ and none had spontaneous activity. In patients, none of the MVRC or frequency ramp parameters correlated with the incidence of spontaneous activity while frequency ramp latency measurement correlated inversely and MVRC parameters positively with MUP duration. There was a positive correlation between MUP amplitude and a few MVRC and frequency ramp amplitude measurements (Table 3).

Table 3. Correlations between quantitative electromyography and MVRC and frequency ramp parameters in patients.

\begin{tabular}{|c|c|c|c|}
\hline \multirow{2}{*}{$\begin{array}{l}\text { MVRC/frequency ramp } \\
\text { parameters }\end{array}$} & \multicolumn{3}{|c|}{ qEMG parameters } \\
\hline & Fibs/psws & MUP duration & MUP amplitude \\
\hline MRRP (ms) & Rho $=0.21$ & $R=-0.17$ & $R=-0.34$ \\
\hline MSuperN $(<15) \%$ & Rho $=-0.11$ & $R=0.52$ & $\mathrm{R}=0.26$ \\
\hline M5SuperN $(<15) \%$ & Rho $=-0.23$ & $\mathbf{R}=\mathbf{0 . 5 7}$ & $\mathrm{R}=0.40$ \\
\hline MLSuperN(50-150)\% & Rho $=-0.32$ & $R=0.49$ & $\mathrm{R}=-0.001$ \\
\hline MXLSuperN(50-150)\% & Rho $=-0.12$ & $\mathbf{R}=\mathbf{0 . 5 1}$ & $\mathrm{R}=0.36$ \\
\hline MX5LSuperN(50-150)\% & Rho $=-0.10$ & $\mathrm{R}=\mathbf{0 . 5 1}$ & $\mathrm{R}=0.47$ \\
\hline Lat $(15 \mathrm{~Hz}) \%$ & $\mathrm{Rho}=0.04$ & $R=\mathbf{- 0 . 5 1}$ & $\mathrm{R}=0.05$ \\
\hline $\operatorname{Latf}(15 \mathrm{~Hz}) \%$ & Rho $=-0.15$ & $R=-0.26$ & $\mathrm{R}=0.16$ \\
\hline $\operatorname{Pkf}(15 \mathrm{~Hz}) \%$ & Rho $=-0.23$ & $\mathrm{R}=0.24$ & $\mathrm{R}=0.60$ \\
\hline $\operatorname{Latf}(30 \mathrm{~Hz}) \%$ & Rho $=-0.11$ & Rho $=-0.23$ & Rho $=-0.14$ \\
\hline $\operatorname{Pkf}(30 \mathrm{~Hz}) \%$ & Rho $=-0.18$ & $\mathrm{R}=0.30$ & $\mathrm{R}=0.47$ \\
\hline
\end{tabular}


$\mathrm{R}$ values were given for parametric tests and Rho for non-parametric correlation analyses depending on the normality of the data. Bold numbers indicate correlations with significant $p$ values. qEMG: Quantitative electromyography, MVRC: Muscle velocity recovery cycle, MRRP: Muscle relative refractory period. MSuperN $(<X)$ : Early supernormality at inter stimulus intervals below $15 \mathrm{~ms}$. M5SuperN: Supernormality with 5 conditioning stimuli. MLSuperN: Late supernormality. MXLSuperN: Extra late supernormality. MX5LSuperN: Extra late supernormality after 5 conditioning stimuli. MRSuperN: Residual Supernormality. MX5RSuperN: Residual supernormality after 5 conditioning stimuli. Lat $(\mathrm{XHz})$ : Latency of last response in train of stimuli at X Hz relative to unconditioned. Latf: Latency of first response in train of stimuli relative to unconditioned. Pkf: Peak of first response in a train of stimuli relative to unconditioned.

None of the MVRC or frequency ramp parameters correlated with the SOFA score. There was a significant positive correlation between the latency from direct muscle stimulation and median nerve CMAP duration $(\mathrm{Rho}=0.65, \mathrm{p}=0.007)$, but not with tibial or peroneal nerve CMAP duration including from anterior tibial muscle. None of the MVRC or frequency ramp parameters correlated with the number of days in ICU on the day of electrophysiological examination or survival (number of survived days from electrophysiological examination).

None of the MVRC or frequency ramp parameters correlated with $\mathrm{K}^{+}, \mathrm{Na}^{+}, \mathrm{Ca}^{2+}, \mathrm{Cl}^{-}$, lactate, creatine, $\mathrm{P}_{\mathrm{a}} \mathrm{CO}_{2}, \mathrm{pH}$, bicarbonate or base excess levels in the blood.

\section{Discussion}

The most striking finding we showed in this study was the high sensitivity of MVRC and frequency ramp parameters (up to $100 \%$ ) in detecting abnormalities in critically ill patients. These tests could detect altered muscle fiber membrane properties in patients with ICUAW which are possibly the first sign of evolving CIM. While myopathic changes (up to 63\%) in qEMG and decrease in CMAP amplitudes (up to 58\%) in NCS were common, the synchronous and smooth long duration CMAPs proposed to be characteristic for CIM (Park et al., 2004; Goodman et al., 2009) were only seen in one patient with severe myopathy out of 24 patients.

\subsection{Conventional EMG and NCS in critically ill patients}


MUP analysis may be challenging in unconscious patients but we could recruit MUPs in all patients in biceps brachii and mostly in anterior tibial muscles with passive movements. qEMG showed in this study myopathic changes in biceps brachii in 15 patients $(63 \%)$ and in anterior tibial muscle in $6(27 \%)$. MVRC and frequency ramp parameters correlated inversely with MUP duration, but these were much more sensitive (up to 100\%) than both MUP duration and amplitude in detecting abnormality.

In contrast to the myopathic changes in the majority of patients, there were only 3 patients (13\%) with polyneuropathy. In all patients with polyneuropathy, there were also signs of myopathy. While there is agreement in literature that CIM is more common than CIP (Lacomis et al., 1998; Trojaborg et al., 2001), the incidence of CIP is controversial (Koch et al., 2011; Crone, 2017). The possible reason for this maybe the difficulty of performing sensory NCS in ICU (Tankisi et al., 2020), and particularly sural nerve may be abnormal due to oedema in these patients. We found reduced SNAP amplitudes both in upper and lower limbs in 3 patients which we interpreted as CIP. However, 2 other patients with oedema in legs had reduced or absent sural SNAPs but normal median and ulnar sensory NCS. Examination of the distal segment of the sural nerve was also normal, therefore we concluded that these patients did not have polyneuropathy. We recommend, examination of distal sural nerve (Tankisi et al., 2019; Kural et al., 2019) in such conditions and that CIP diagnosis is not only based on sural nerve but also on upper extremity sensory nerves. The incidence of myopathy in our study was high but polyneuropathy was rare. This is consistent with two previous studies (Trojaborg et al., 2001; Crone, 2017), although in the study by Crone, in only 20 out of 49 patients were sural NCS interpreted as technically satisfactory and included in the analysis. Of the 20 patients, in only 4 subjects CIP diagnosis was given after excluding the patients with a possible aetiology for neuropathy. None of our patients had a neuropathy or myopathy diagnosis before admission to ICU but we did not exclude the patients with possible underlying causes for 
polyneuropathy. We propose that the only possible way to explore the true incidence of CIP is to do longitudinal studies in ICU starting from the day the patients are admitted to ICU until the day of discharge.

Prolongation in distal CMAP duration without marked desynchronization has been proposed as a characteristic sign of CIM (Cros, 1997; Trojaborg, 2006). However, the existing literature on the utility of CMAP duration in CIM is limited to retrospective studies (Kramer et al., 2017) and short cohorts of even less than 10 patients (Park et al., 2004). In a later study by Trojaborg (Trojaborg et al., 2006), the high sensitivity of CMAP prolongation reported by Park et al. could not be confirmed. We had also only one patient with prominent increase in CMAP duration with this configuration who had severe myopathic changes. We propose that this finding is a sign of severe myopathy and using this for differential diagnosis may be misleading in milder conditions. This feature with CMAP prolongation has been attributed to be due to slowing of muscle fiber conduction velocities as a result of blockage of voltage gated $\mathrm{Na}^{+}$channels in the muscle fibers (Allen et al., 2008). Loss of $\mathrm{Na}^{+}$channel function in CIM, either directly via some unknown cellular signalling or indirectly via depolarization of the muscle fibres, is consistent with the increase in relative refractory period observed here. Our finding of correlation between the latency of direct muscle stimulation evoked potential and CMAP duration further supports this hypothesis. However, in the only patient in whom we found characteristic CMAP prolongation, the latency to the direct muscle stimulation was severely prolonged, but interestingly MVRC changes were less pronounced than in other patients. In this patient, profuse fibs/psws were observed which may suggest denervated intramuscular nerves rather than ion channel dysfunction in the pathogenesis of CMAP prolongation.

In spite of normal CMAP duration, we found in most patients reduced CMAP amplitudes and absent F-waves, which might have caused in previous cohorts misinterpretation in favour of 
polyneuropathy. In two recent case reports, the initial lack of F-waves in CIM patients could be reverted after $20 \mathrm{~Hz}$ repetitive nerve stimulation (Zimnowodzki et al., 2020; Tankisi et al., 2020c). This wake-up phenomenon may suggest decreased motor neuron excitability, which is possibly due to a prolonged period of reduced mobility. In the present study, we did not perform $20 \mathrm{~Hz}$ repetitive nerve stimulation, but future studies should include this simple procedure in the examination protocol.

Another electrophysiological parameter which is also included in the diagnostic criteria (Latronico and Bolton, 2011) is neCMAP/mCMAP ratio. A ratio $>0.5$ has been proposed as a characteristic sign of CIM, but it is not sensitive or specific enough to diagnose CIM: this ratio cannot distinguish CIM from a healthy muscle, and it cannot differentiate neuromyopathy as also seen in our patients.

\subsection{MVRCs as a potential biomarker for CIM?}

To date, MVRCs have been used in one previous study in 10 patients with CIM. We wanted to take this study further, using more advanced MVRC protocols in a larger cohort. We found similar changes to the previous study by Z'Graggen and coworkers, with prolonged MRRP and reduced early and late supernormality. We found the most prominent changes with 5 conditioning stimuli and the frequency ramp: protocols that were not included in the study by Z'Graggen et al. (2011). There was abnormality in one or more parameters in all 24 patients, suggesting MVRCs as a sensitive test to detect changes in critically ill patients and therefore a potential biomarker for ICUAW. One should keep in mind, however, that these changes are not specific for CIM, and that MVRC measurements cannot distinguish between membrane depolarization and an increase in sodium channel inactivation at the same membrane potential. We found similar changes as in ischemia (ZGraggen and Bostock, 2009), renal insufficiency (Z’Graggen et al., 2010), inclusion body myositis (Lee et al., 2019) and spinal cord injury (Witt et al., 2020). Additionally, we have shown in a recent study that neurogenic muscles have similarly altered muscle fiber membrane 
properties (Witt et al., 2019). In neurogenic muscles, MVRC parameters correlated significantly to the incidence of fibs/psws, which we have not seen in the present study. We have only seen fibs/psws in 4 patients, despite abnormal MVRC properties in all patients. This may suggest that different cellular mechanisms are responsible for the altered muscle fiber membrane properties revealed by MVRCs in critically ill patients and in other conditions.

MVRCs revealed prolonged refractory period and slowed conduction velocity of action potentials in the muscle of CIM patients. These findings are likely to reflect altered function of the voltage gated sodium channels in skeletal muscle fibers (Nav1.4). At the cellular level, the action potential upstroke is created by inward $\mathrm{Na}^{+}$current through $\mathrm{Nav} 1.4$ and it is this ionic current that invades resting membrane in front of the action potential waveform to propagate the action potential. Prolonged refractory period and slowed conduction velocity would be expected to arise if excessive Nav1.4 inactivation or depolarized shift in the activation voltage of Nav1.4 had evolved. Such changes in NaV1.4 function could be envisaged to take place in response to some unknown cellular signaling system involved in CIM, or they could happen secondary to chronic depolarization of the muscle fiber membrane in CIM. Depolarization can, in turn, arise due to a number of different cellular changes such as Nav1.4 leak, loss of $\mathrm{Na}^{+}, \mathrm{K}^{+}$-ATPase activity or loss of $\mathrm{K}^{+}$channel function. It could also arise due to excessive loss of $\mathrm{K}^{+}$from the intracellular space to the extracellular space. Z’Graggen and coworkers found in a previous study (Z’Graggen et al., 2011) significant correlation between MVRC parameters and plasma $\mathrm{K}+$ levels, although the plasma $\mathrm{K}+$ levels were in normal ranges, which speaks against possible muscle membrane depolarization by hyperkalemia as in chronic renal failure (Z'Graggen et al., 2010). We hypothesized that we would find even more pronounced relationships in this study between plasma $\mathrm{K}^{+}$levels and early and late supernormality with 5 conditioning stimuli and frequency ramp measures, but we did not find any correlation between the muscle excitability parameters and plasma $\mathrm{K}^{+}$or the other electrolytes. We 
believe this may be due the local rules in management of $\mathrm{K}^{+}$levels in blood, since we found very small fluctuations in $\mathrm{K}^{+}$levels. Another difference is that we used anterior tibial muscle whereas Z'Graggen and coworkers did the recording in brachioradialis muscle, but we think that is unlikely to explain the different findings.

So while MVRC clearly has the highest sensitivity and specificity for detecting altered membrane properties of skeletal muscle fibers in CIM patients, the cellular mechanism that underlie the loss of excitability in CIM will need further study, and to what extent CIM patients have depolarized muscle fibers must be determined directly using intracellular microelectrodes.

\subsection{Limitations}

The main limitation of this study is the small number of patients, but this number was comparable with the literature. Additionally, the mean age of the of our patients was higher than the control group, although the difference was not significant. Taking into account that a previous study showed a strong correlation of depolarised appearances in MVRC with age (Lee et al., 2018) some of our results may be influenced by this age difference. However, we do not think that age can fully account for the pronounced changes in this study. Another limitation is the lack of muscle biopsies, so that we could only make a diagnosis of probable CIM. Additionally, the duration of ICU stay was very variable. Moreover, it would be ideal to do the examinations in a proximal muscle. Although anterior tibial muscle, with a well-defined end-plate zone, is the most commonly used muscle for MVRC recordings, peroneal nerve entrapment in ICU may be expected, which could have influenced our results. We attempted to exclude this by NCS of the segments across the knee, and the low incidence of spontaneous activity, myopathic MUPs and the neCMAP/mCMAP ratios $>0.5$ argue against an entrapment neuropathy. One more limitation of this study is that we did not perform muscle ultrasound, a method that is gaining popularity for showing particularly muscle mass (Paris et al., 2017). Since electrophysiological studies can provide information about function, 
which is not possible with ultrasound, further studies are necessary to explore whether muscle ultrasound can be a supplementary test to the conventional and novel electrophysiological methods. Additionally, we acknowledge that, whereas conventional NCS and EMG should be accessible in every hospital, the newer technique of MVRC recording is not available in most ICUs.

\subsection{Conclusion}

MVRCs provide a useful supplementary test to conventional NCS and EMG for the diagnosis of CIM. In addition to diagnosis, such clinical tools could further facilitate better understanding of disease mechanisms in CIM. In contrast to previous studies, we found synchronous and smooth long duration CMAPs only as a sign of severe CIM. While myopathy is common in ICUAW, polyneuropathy is rare, but cannot be excluded as due to already existing neuropathy. Longitudinal studies in larger cohorts will be needed to determine the incidence of CIM and CIP.

\section{References:}

Allen DC, Arunachalam R, Mills KR. Critical illness myopathy: further evidence from muscle-fiber excitability studies of an acquired channelopathy. Muscle Nerve 2008;37:14 -22.

Boland-Freitas R, Lee J, Howells J, Liang C, Corbett A, Nicholson G, Ng K. Sarcolemmal excitability in the myotonic dystrophies. Muscle Nerve 2018 Apr;57(4):595-602.

Boles JM, Bion J, Connors A, Herridge M, Marsh B, Melot C, Pearl R, Silverman H, Stanchina M, Vieillard-Baron A, Welte T. Weaning from mechanical ventilation. Eur Respir J 2007;29:10331056.

Bolton CF. Neuromuscular manifestations of critical illness. Muscle Nerve 2005;32:140-163.

Buchthal F. The electromyogram: its value in the diagnosis of neuromuscular disorders. World Neurol 1962;3:16-34. 
Crone C. Tetraparetic critically ill patients show electrophysiological signs of myopathy. Muscle Nerve 2017;56(3):433-440.

Friedrich O, Reid MB, Van den Berghe G, Vanhorebeek I, Hermans G, Rich MM, Larsson L. The Sick and the Weak: Neuropathies/Myopathies in the Critically Ill. Physiol Rev 2015 Jul;95(3):1025109.

Goodman BP, Harper CM, Boon AJ. Prolonged compound muscle action potential duration in critical illness myopathy. Muscle Nerve 2009;40:1040-1042.

Koch S, Spuler S, Deja M, Bierbrauer J, Dimroth A, Behse F, et al. Critical illness myopathy is frequent: accompanying neuropathy protracts ICU discharge. J Neurol Neurosurg Psychiatry 2011;82:287-293

Koch S, Wollersheim T, Bierbrauer J, Haas K, Mörgeli R, Deja M, et al. Long-term recovery in critical illness myopathy is complete, contrary to polyneuropathy. Muscle Nerve 2014;50:431-436

Kural MA, Karlsson P, Pugdahl K, Isak B, Fuglsang-Frederiksen A, Tankisi H. Diagnostic utility of distal nerve conduction studies and sural near-nerve needle recording in polyneuropathy. Clin Neurophysiol 2017;128(9):1590-1595.

Lacomis D, Petrella JT, Giuliani MJ. Causes of neuromuscular weakness in the intensive care unit: a study of ninety-two patients. Muscle Nerve. 1998 May;21(5):610-7.

Latronico N, Bolton CF. Critical illness polyneuropathy and myopathy: a major cause of muscle weakness and paralysis. Lancet Neurol 2011;10:931-941.

Lee JHF, Boland-Freitas R, Ng K. Sarcolemmal excitability changes in normal human aging. Muscle Nerve 2018;57(6):981-988.

Lee JH, Boland-Freitas R, Liang C, Ng K. Sarcolemmal depolarization in sporadic inclusion body myositis assessed with muscle velocity recovery cycles. Clin Neurophysiol 2019;130(12):22722281.

Llano-Diez M, Renaud G, Andersson M, Marrero HG, Cacciani N, Engquist H, et al. Mechanisms underlying ICU muscle wasting and effects of passive mechanical loading. Crit Care 2012;16(5):R209.

Maggiore SM, Battilana M, Serano L, Petrini F. Ventilatory support after extubation in critically ill patients. Lancet Respir Med 2018;6(12):948-962.

Moreno RP, Metnitz PG, Almeida E, Jordan B, Bauer P, Campos RA, et al.; SAPS 3 Investigators. SAPS 3--From evaluation of the patient to evaluation of the intensive care unit. Part 2:

Development of a prognostic model for hospital mortality at ICU admission. Intensive Care Med 2005;31(10):1345-55.

Paris MT, Mourtzakis M, Day A, Leung R, Watharkar S, Kozar R, et al. Validation of Bedside Ultrasound of Muscle Layer Thickness of the Quadriceps in the Critically Ill Patient (VALIDUM Study). JPEN J Parenter Enteral Nutr. 2017;41(2):171-180. 
Park EJ, Nishida T, Sufit RL, Minieka MM. Prolonged compound muscle action potential duration in critical illness myopathy: report of nine cases. J Clin Neuromuscul Dis 2004;5:176-183.

Rich MM, Bird SJ, Raps EC, McCluskey LF, Teener JW. Direct muscle stimulation in acute quadriplegic myopathy. Muscle Nerve 1997;20:665-673.

Stålberg E, van Dijk H, Falck B, Kimura J, Neuwirth C, Pitt M, et al. Standards for quantification of EMG and neurography. Clin Neurophysiol 2019;130(9):1688-1729.

Tan SV, Suetterlin K, Männikkö R, Matthews E, Hanna MG, Bostock H. In vivo assessment of interictal sarcolemmal membrane properties in hypokalaemic and hyperkalaemic periodic paralysis. Clin Neurophysiol 2020;131(4):816-827.

Tan SV, Z'Graggen WJ, Hanna MG, Bostock H. In vivo assessment of muscle membrane properties in the sodium channel myotonias. Muscle Nerve 2018;57(4):586-594.

Tankisi H, Pugdahl K, Johnsen B, Fuglsang-Frederiksen A. Correlations of nerve conduction measures in axonal and demyelinating polyneuropathies. Clin Neurophysiol 2007;118(11):2383-92.

Tankisi H, Pugdahl K, Beniczky S, Andersen H, Fuglsang-Frederiksen A. Evidence-based recommendations for examination and diagnostic strategies of polyneuropathy electrodiagnosis. Clin Neurophysiol Pract 2019;4:214-222.

Tankisi H, de Carvalho M, Z'Graggen WJ. Critical Illness Neuropathy. J Clin Neurophysiol 2020a;37: 205-207.

Tankisi H, Burke D, Cui L, de Carvalho M, Kuwabara S, Nandedkar SD, et al. Standards of instrumentation of EMG. Clin Neurophysiol 2020b;131(1):243-258.

Tankisi H, Tankisi A, Harbo T, Markvardsen LK, Andersen H, Pedersen TH. Critical illness myopathy as a consequence of Covid-19 infection. Clin Neurophysiol. 2020c Aug;131(8):19311932.

Trojaborg W, Weimer LH, Hays AP. Electrophysiologic studies in critical illness associated weakness: myopathy or neuropathy--a reappraisal. Clin Neurophysiol 2001;112(9):1586-93.

Trojaborg W. Electrophysiologic techniques in critical illness-associated weakness. J Neurol Sci 2006;242(1-2):83-5.

Vincent JL, Ferreira F, Moreno R. Scoring systems for assessing organ dysfunction and survival. Crit Care Clin 2000;16(2):353-66.

Witt A, Kristensen RS, Fuglsang-Frederiksen A, Pedersen TH, Finnerup NB, Kasch H, Tankisi H. Muscle velocity recovery cycles in neurogenic muscles. Clin Neurophysiol 2019;130(9):1520-1527.

Witt A, Fuglsang-Frederiksen A, Finnerup NB, Kasch H, Tankisi H. Detecting peripheral motor nervous system involvement in chronic spinal cord injury using two novel methods: MScanFit MUNE and muscle velocity recovery cycles. Clin Neurophysiol 2020;131(10):2383-2392. 
Z’Graggen WJ, Tankisi H. Critical Illness Myopathy. J Clin Neurophysiol 2020;37: 200-204.

Z'Graggen WJ, Bostock H. Velocity recovery cycles of human muscle action potentials and their sensitivity to ischemia. Muscle Nerve 2009;39:616-626.

Z'Graggen WJ, Aregger F, Farese S, Humm AM, Baumann C, Uehlinger DE, Bostock H. Velocity recovery cycles of human muscle action potentials in chronic renal failure. Clin Neurophysiol 2010;121(6):874-81.

Z'Graggen WJ, Brander L, Tuchscherer D, Scheidegger O, Takala J, Bostock H: Muscle membrane dysfunction in critical illness myopathy assessed by velocity recovery cycles. Clin Neurophysiol 2011;122:834-841.

Zimnowodzki S, Butrum M, Kimura J, Stålberg E, Mahajan S, Gao L. Emergence of F-waves after repetitive nerve stimulation. Clin Neurophysiol Pract. 2020 May 8;5:100-103.

Witt A, Bostock H, Z'Graggen WJ, Tan SV, Kristensen AG, Kristensen RS, Larsen LH, Zeppelin Z, Tankisi H. Muscle Velocity Recovery Cycles to Examine Muscle Membrane Properties. J Vis Exp. 2020 Feb 19;(156). 


\section{Figure legends:}

Figure 1. Quantitative electromyography of the biceps brachii muscle excited by passive movements of the elbow by the examiner in a sedated patient with critical illness myopathy (A) and in a healthy control (B). There are myopathic motor unit potentials with decreased amplitude and short duration in the patient and normal motor unit potentials in the healthy subject.

Figure 2. a) Synchronous and smooth long duration compound muscle action potentials (CMAPs) in a patient with severe myopathy (patient \#19) (A) and normal duration in another patient with moderate myopathy (patient \#17) (B). CMAP amplitude is decreased but distal motor latency (DML) and conduction velocity (CV) at forearm are in normal limits in both patients. Electromyography showed profuse fibrillations and positive sharp waves in patient \#19 while there was no spontaneous activity in patient \#17. b) Muscle velocity recovery cycles (MVRCs) of patient \#19 (lines) on the left compared to the mean of healthy controls (open circles). Red indicate MVRCs after one, green 2 and blue 5 conditioning stimuli. On the right, severely abnormal MVRCs in a patient with moderate myopathy is shown.

Figure 3. a) Dot plots of the muscle velocity recovery cycles (MVRCs) and quantitative electromyography measurements in patients compared to healthy subjects. A: Muscle relative refractory period (MRRP). B: Late supernormality (MX5LSuperN(50-150)\%) after 5 conditioning stimuli. C: Motor unit potential (MUP) duration. D: Motor unit potential amplitude. Solid lines are the mean of the group, dashed lines are $95 \%$ confidence limits for the control group. Asterisks indicate level of significance $(* * *=p<0.001$, $* * * * *=p<0.00001)$. b) ROC curves describing the ability of muscle velocity recovery cycles (MVRCs) and frequency ramp measures and motor unit 
potential (MUP) duration and amplitude to discriminate healthy controls from patients in anterior tibial muscle.

Figure 4. a) Muscle velocity recovery cycles (MVRCs) following 1 (left), 2 (middle) and 5 (right) conditioning stimuli, recorded from healthy controls (green) and patients (red lines). Green solid lines are the mean of the group, dashed lines indicate $95 \%$ confidence intervals. b) Frequency ramp responses. Top: Latency changes produced by increasing frequency from 1 to $30 \mathrm{~Hz}$ for 1 minute every 2 minutes. Latency changes were recorded to the first and last stimulus in each train, compared with the control values. Middle: The corresponding changes in peak amplitude. Bottom: Frequency during the trains. Healthy controls are depicted in green and patients in red. 
A

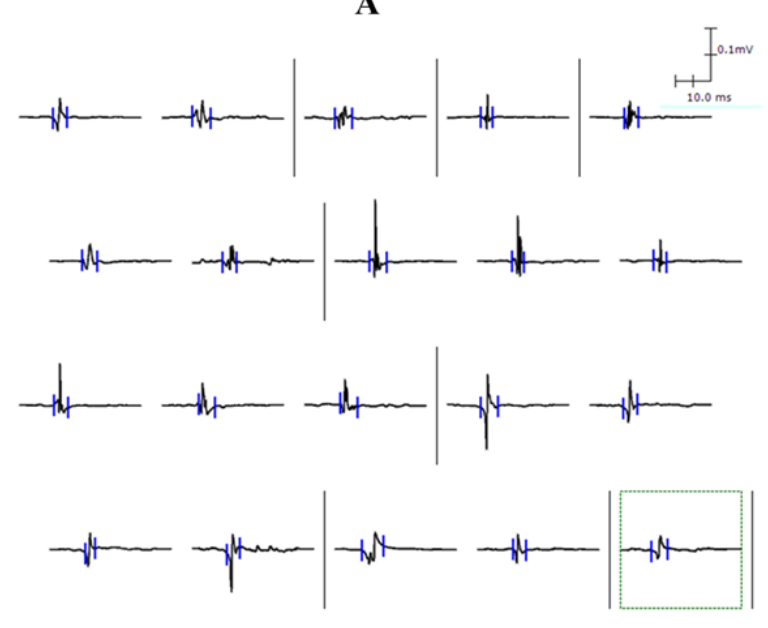

B

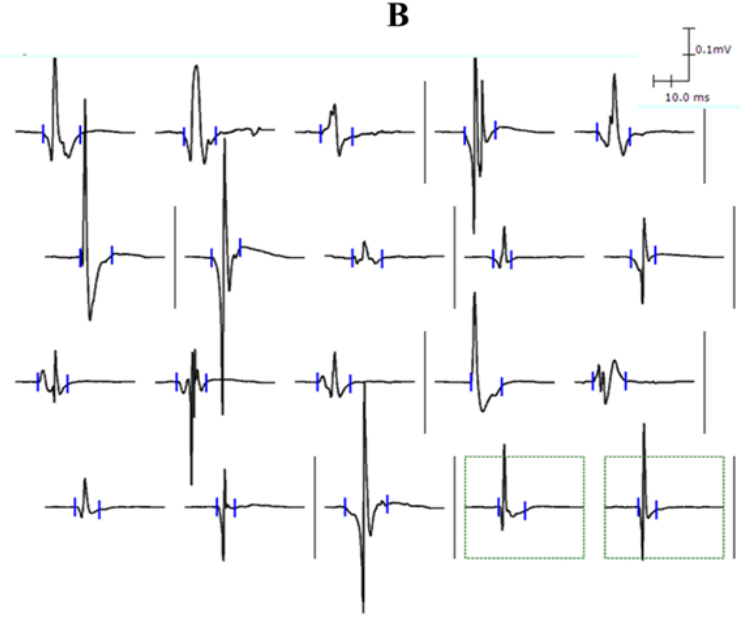

Figure 1. 
A

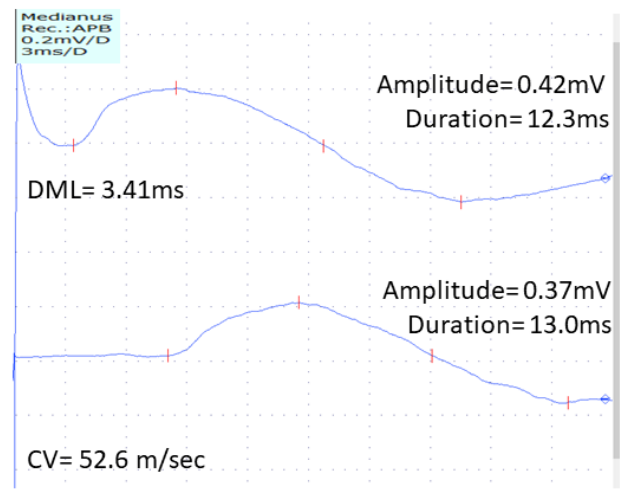

B

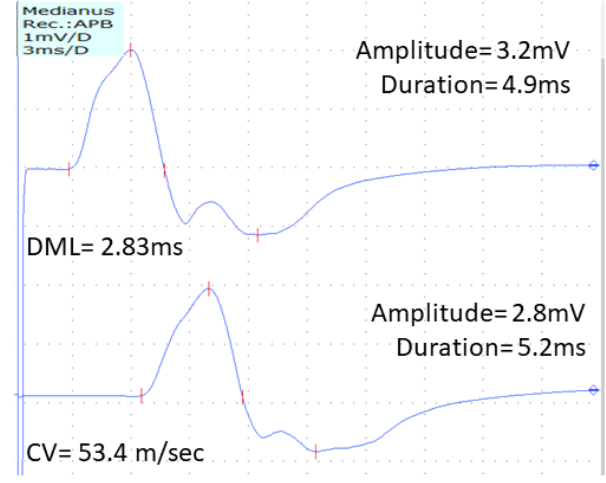

Figure 2a. 

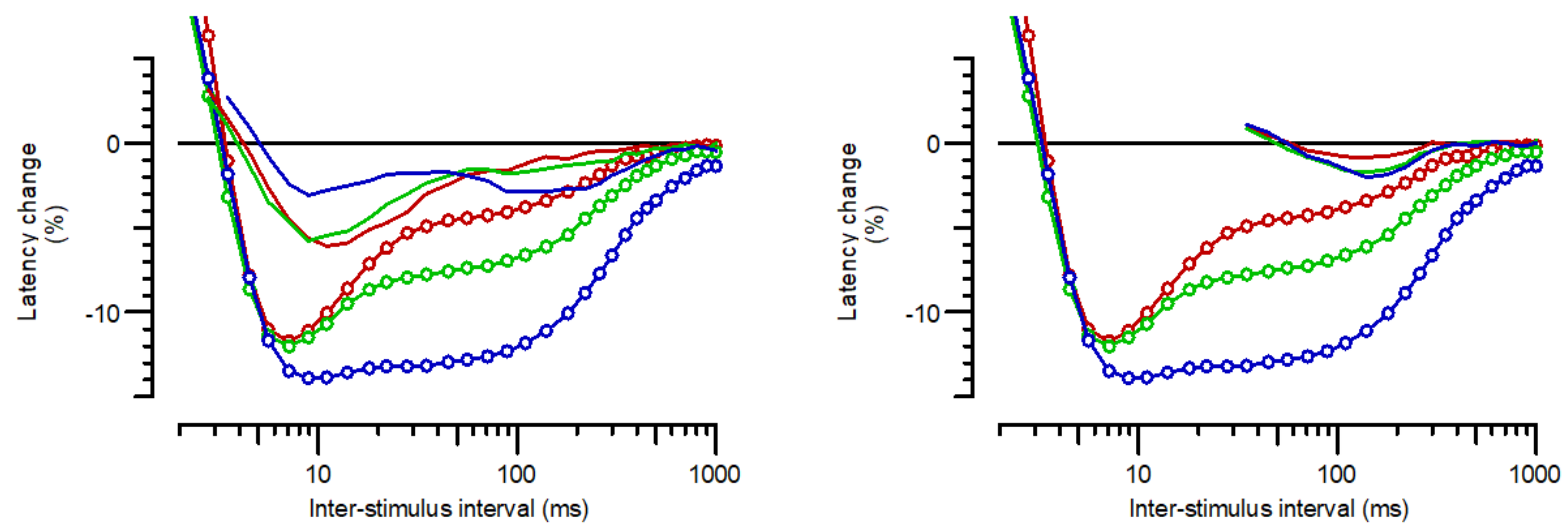

Figure 2b 

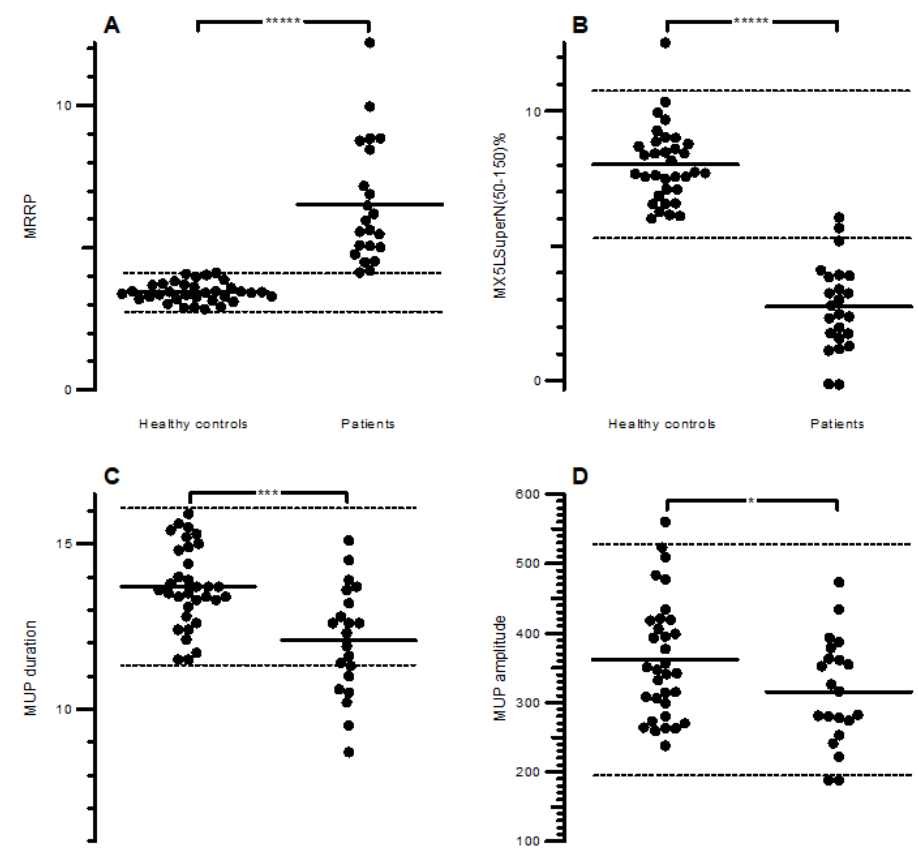

Figure 3a 


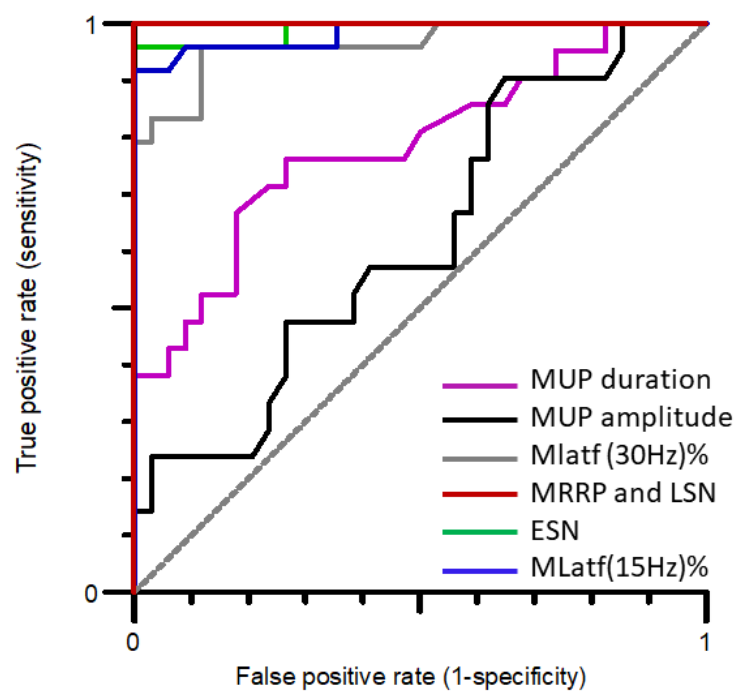

Figure 3b 

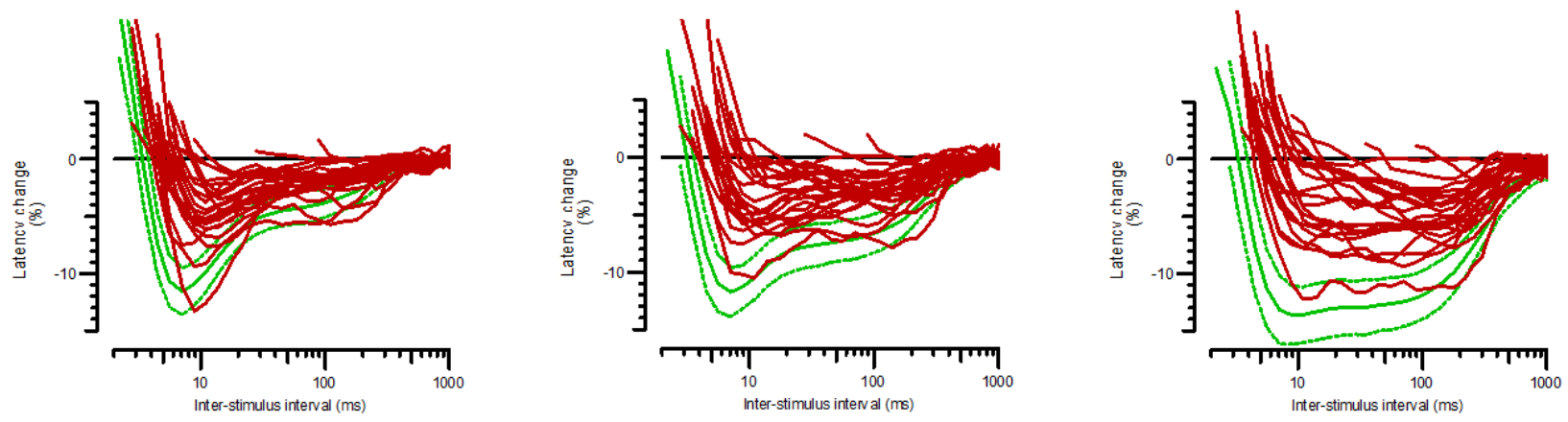

Figure 4a 

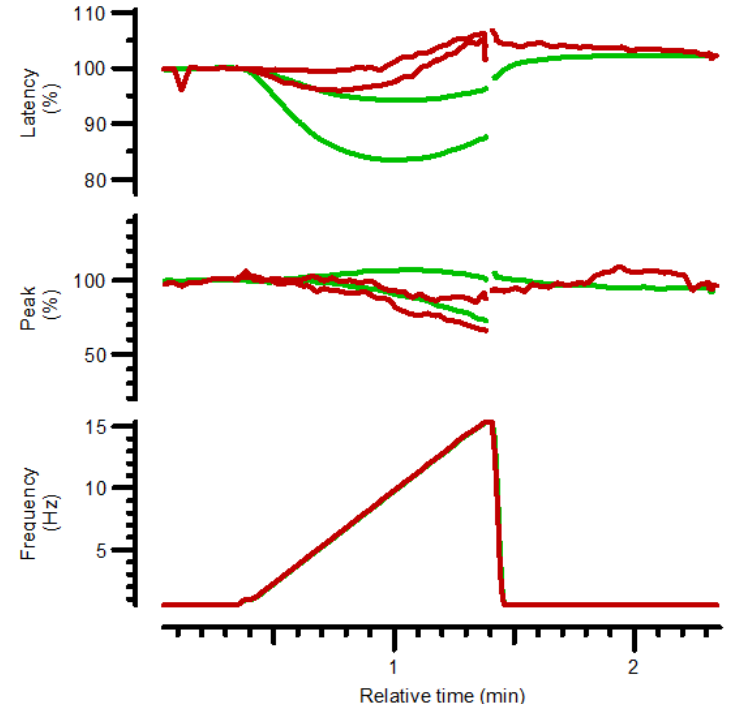

Figure 4b 